DOI: $10.7242 / 2658-705 X / 2019.1 .2$

УДК 532.72, 537.84, 537.621

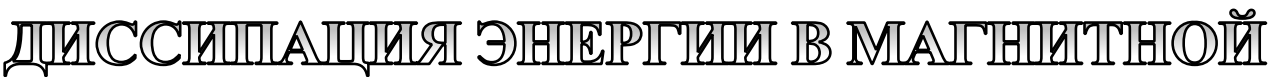

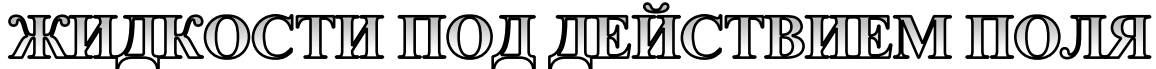 3IBIIROBBOIZ̆ पIACTIOTII**
}

М.А. Косков, Институт механики сплошных сред УрО РАН

Пермский государственный национальный исследовательский университет

А.Ф. Пшеничников, Институт механики сплошных сред УрО РАН

Пермский государственный национальный исследовательский университет

Экспериментально исследована диссипация энергии в магнитной жидкости, находящейся в переменном магнитном поле звуковой частоты. Цель работы получение информации об амплитудной зависимости динамической восприимчивости и границах применимости линейного релаксационного уравнения для намагниченности. В эксперименте использовалась очищенная от крупнодисперсной фрракции, методом центрифугирования, магнитная жидкость. Проведены измерения тепловыделения в магнитной жидкости при повышенных амплитудах магнитного поля. Использовалась известная формула, связывающая мощность тепловыделения с мнимой частью восприимчивости и непосредственно вытекающая из линейного релаксационного уравнения. Контрольные прямые измерения динамической восприимчивости проводились методом взаимной индуктивности в слабом зондирующем поле при различных значениях температуры магнитной жидкости. Сопоставление результатов, полученных разными методами, не выявило существенного расхождения между двумя сериями опытов, что объясняется существенным преобладанием в растворе частиц, для которых параметр Ланжевена остаётся малым при повышенных амплитудах зондирующего поля. Как результат, динамическая восприимчивость остаётся квазинезависимой от напряжённости поля по крайней мере до 1,3 кА/м.

Ключевые слова: переменное магнитное поле, диссипация энергии, магнитная жидкость, динамическая восприимчивость.

Магнитные жидкости - коллоидные растворы ферромагнитных частиц в жидкости-носителе - представляют собой суперпарамагнитные системы $[10,14]$. Если жидкость находится в состоянии близком к термодинамическому равновесию, динамика намагниченности М вполне корректно описы- вается линейным релаксационным уравнением $[1,9,10]$.

$$
\frac{d \mathbf{M}}{d t}=-\frac{1}{\tau}\left(\mathbf{M}-\mathbf{M}_{0}\right)
$$

где $\mathbf{M}_{0}$ - равновесная намагниченность, $\tau$ - характерное время релаксации. В слабых полях равновесная намагниченность прямо пропорциональна напряжённости

* Работа выполнена при финансовой поддержке РФФИ (проект № 16-01-00517). 
$\left(\mathrm{M}_{0}=x_{0} \mathrm{H}, x_{0}-\right.$ начальная восприимчивость), а вследствие линейности уравнения (1) динамическая намагниченность так же пропорциональна напряжённости магнитного поля. В случае переменного линейно-поляризованного поля $H=H_{0} \mathrm{e}^{i \omega t}$, решение уравнения (1) имеет вид

$$
\tilde{M}=\left(x_{1}-i x_{2}\right) H_{0} e^{i \omega t},
$$

где действительная и мнимая части динамической восприимчивости описываются формулами Дебая [10, 14]

$$
x_{1}=\frac{x_{0}}{1+(\omega \tau)^{2}}, \quad x_{2}=\frac{x_{0} \omega \tau}{1+(\omega \tau)^{2}} .
$$

Мнимая часть восприимчивости $x_{2}$ отвечает за диссипацию энергии магнитного поля в жидкости, происходящую вследствие чередующихся процессов перемагничивания. Согласно [14] мощность, рассеиваемая в некотором объёме $V$ магнитной жидкости, находящейся в слабом переменном магнитном поле, определяется по формуле

$$
Q=\mu_{0} \omega x_{2} V \frac{H_{0}^{2}}{2},
$$

Где $\mu_{0}=4 \pi \cdot 10^{-7} \quad$ Гн/м - магнитная постоянная вакуума, $\mathrm{H}_{0}$ и $\omega$ - амплитуда и циклическая частота магнитного поля соответственно.

Реальные магнитные жидкости обладают широким спектром времён релаксации, связанным с полидисперсностью однодоменных частиц, существованием кластеров и двух независимых механизмов релаксации: броуновским и неелевским. Вследствие этого формулы (2), соответствующие системе с одним временем релаксации, трансформируются в ряды по функциям Дебая:

$$
\begin{aligned}
& \chi_{1}(\omega)=A_{0}+\sum_{i=1}^{N} \frac{A_{i} \omega \tau_{i}}{1+\left(\omega \tau_{i}\right)^{2}}, \\
& \chi_{2}=\sum_{i=1}^{N} \frac{A_{i} \omega \tau_{i}}{1+\left(\omega \tau_{i}\right)^{2}} .
\end{aligned}
$$

Интересующая нас низкочастотная часть спектра восприимчивости хорошо описывается относительно небольшим
$(N=4 \ldots 7)$ числом слагаемых, каждое из которых соответствует отдельной фракции частиц с фиксированным временем релаксации $\tau_{\mathrm{i}} \quad[3,6] . \quad$ Спектральные амплитуды $A_{i}$ показывают вклад $i$-й фракции частиц в начальную восприимчивость. $A_{0}$ описывает частотно независимый (до $10^{5}$ Гц) вклад самых мелких частиц с неелевским механизмом релаксации. Сумма всех спектральных амплитуд, очевидно, представляет собой начальную восприимчивость. Если фракция представлена отдельными частицами, спектральные амплитуды можно описать в рамках модифицированной модели эффективного поля [1].

$$
A_{i} \approx \frac{\mu_{0}\left\langle m_{i}^{2}\right\rangle n_{i}}{3 k T} \cdot\left(1+\frac{\chi_{L}}{3}\right) \quad \chi_{L}=\frac{\mu_{0}\left\langle m^{2}\right\rangle n}{3 k T},
$$

где $n$ и $n_{i}$ - числовые плотности для системы в целом и выделенной фракции соответственно, $m$ - магнитный момент частицы. Угловые скобки означают усреднение по ансамблю.

В пределе низких частот квазиравновесная восприимчивость не зависит от амплитуды, когда параметр Ланжевена, соответствующий самым крупным частицам, достаточно мал. Если допускать погрешность порядка одного процента, то соответствующее условие имеет вид

$$
\xi=\frac{\mu_{0} m H_{0}}{k T} \leq 0,3 .
$$

Для типичных магнетитовых коллоидов это условие выполняется при амплитуде поля в пределах 400-500 A/м [7] и является условием выполнимости формул (5) для спектральных амплитуд. В динамическом режиме $\left(\omega \tau_{i} \sim 1\right)$ ключевую роль начинают играть времена релаксации и их зависимость от напряжённости поля и межчастичных взаимодействий, в том числе образование агрегатов. Хотя в последнее время появился ряд работ по влиянию межчастичных взаимодействий на динамику намагниченности, проблема ещё далека от решения [12]. 
Цель данной работы - экспериментальная оценка диапазона зондирующих полей, при которых динамическая восприимчивость может считаться не зависящей от амплитуды поля величиной, а формулы (3-4) могут быть использованы для расчёта диссипации энергии в магнитной жидкости. В прикладном плане работа может представлять интерес в связи с продолжающимися попытками использовать магнитные наночастицы для гипертермии опухолей $[2,4]$. В частности, в [2] обозначены проблемы, связанные с продолжительностью лечебной процедуры и контролем температуры опухоли. Решение обеих проблем требует корректной оценки мощности тепловыделения, подбора частоты и амплитуды поля.

В качестве источника магнитного поля в эксперименте использовался соленоид длиной $L=374$ мм и диаметром $D=33$ мм $(L \gg D)$. Соленоид наматывался эмалированным проводом ПЭВ диаметром 0,31 мм на трубу из поливинилхлорида. Количество витков $N=1080$. В средней части соленоида, на его оси, размещалась пробирка для магнитной жидкости. Длина пробирки 105 мм, внутренний диаметр 8,5 мм. На концах пробирки укреплялись стеклотекстолитовые шайбы, совпадающие по диаметру с внутренним диаметром трубы. Таким образом исключался сдвиг пробирки внутри соленоида в процессе измерений. Для измерения силы тока в соленоиде последовательно к нему подключался безындуктивный резистор $R=1,05$ Ом, средневыпрямленное напряжение на котором измерялось вольтметром Ф5053. Соленоид подключался к усилителю звуковой частоты $100 \mathrm{У}-101$, на который подавался синусоидальный сигнал от низкочастотного генератора ГЗ-112/1.

Магнитное поле на оси соленоида можно вычислить, разбив соленоид на тонкие кольца и пользуясь принципом суперпозиции. Одиночное кольцо толщиной $\Delta z$ создаёт поле напряжённостью

$$
\Delta H=\frac{I H}{L} \frac{D^{2}}{\left(4 z^{2}+D^{2}\right)^{\frac{3}{2}}} \Delta z,
$$

где $I$ - сила тока в витках соленоида, $z$ - расстояние от центра кольца до произвольной точки $Z_{1}$ оси соленоида в которой оценивается напряжённость. Начало координат находится у нижнего торца соленоида (рис. 1). Суммируя вклады отдельных колец и переходя к интегралу по длине соленоида, получаем распределение напряжённости магнитного поля вдоль его оси

$$
H=\frac{I N}{L}\left(\frac{Z_{1}}{\sqrt{4 Z_{1}^{2}+D^{2}}}+\frac{L-Z_{1}}{\sqrt{4\left(L-Z_{1}\right)^{2}+D^{2}}}\right) .
$$

В средней части соленоида формула (7) предсказывает существование области с однородным полем $H=I N / L$. В частности, при указанной выше геометрии соленоида напряжённость поля в месте размещения пробирки достаточно корректно описывалась формулой $H=\alpha I$, $\alpha=(2,88 \pm 0,02) \cdot 10^{3} \mathrm{~m}^{-1}, \quad$ а неоднородность поля не превышала $1 \%$.

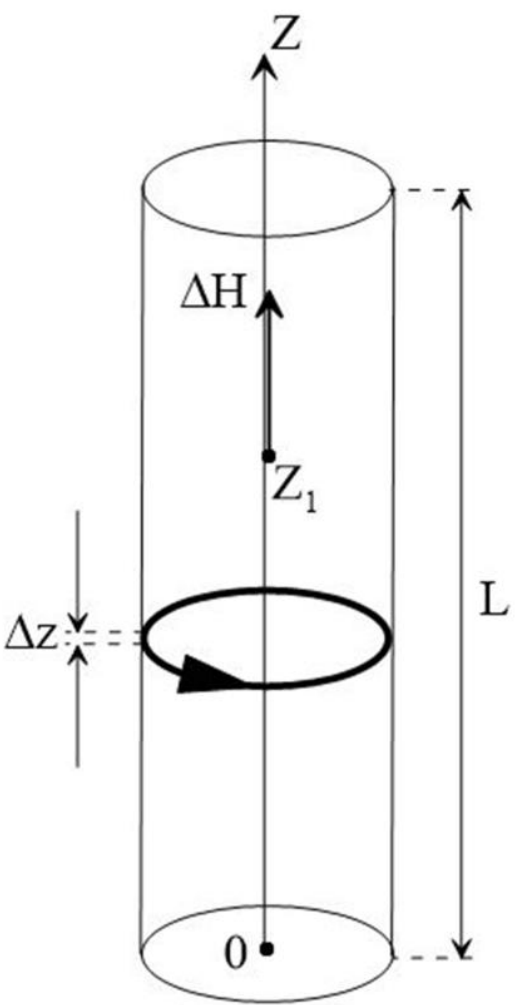

Рис. 1. Схема соленоида и система координат 
В эксперименте использовалась магнитная жидкость на основе керосина и магнетита, стабилизированная олеиновой кислотой. Предварительно, наиболее крупные частицы и агрегаты из жидкости удалялись центрифугированием. Плотность полученной после удаления крупнодисперсной фракции жидкости измерялась пикнометром при комнатной температуре и составила $\rho=1,53 \Gamma / \mathrm{cm}^{3}$. Объёмная доля твёрдой фазы составила $\phi=0,17$ и оценивалась по формуле

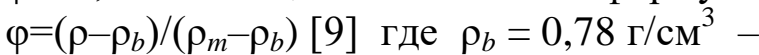
плотность керосина при комнатной температуре, $\rho_{m}=5,2$ г/ $\mathrm{cm}^{3}$ - плотность магнетита. Вязкость жидкости $\eta=2,7 \cdot 10^{-}$ ${ }^{3}$ Па.с оценивалась по формуле Чоу [1]:

$$
\frac{\eta}{\eta_{0}}=\exp \left(\frac{2,5 \varphi}{1-\varphi}\right)+\frac{A \varphi^{2}}{1-A P \varphi^{2}},
$$

где $A=4,67, P=0,605$ - коэффициент упаковки частиц, при котором жидкость теряет текучесть, $\eta_{0}=1,5 \cdot 10^{-3}$ Па·с - вязкость керосина при комнатной температуре. Диапазон рабочих частот определялся из условия $\omega \tau_{B} \sim 1$, где $\tau_{B}=\pi \eta d^{3} /(2 k T)$ - броуновское время релаксации магнитных моментов, соответствующее преобладающей в растворе фракции частиц с гидродинамическим диаметром $d=15$ нм [3]. Оценка броуновского времени релаксации даёт по порядку величины $\sim 10^{-5} \mathrm{c}$, чему соответствуют частоты зондирующего поля 8-16 кГц. Для этого диапазона динамические эффекты должны быть выражены достаточно заметно.

Наиболее сложной частью измерений было нахождение тепловой мощности $Q$, выделяемой магнитной жидкостью. Она определялась по перепаду температур в зазоре между стенкой пробирки и стенкой соленоида. Измерение перепада температуры проводилось при помощи медь-константановой термопары, горячий спай которой приклеивался к стенке пробирки, а холодный - к внутренней стенке соленоида. ЭДС термопары измерялась вольтметром Щ300. Зависимость $\varepsilon(Q)$ между ЭДС термопары $\varepsilon$ и мощностью $Q$ устанавливалась в градуировочных опытах. С этой целью был изготовлен электронагреватель, размеры которого позволяли разместить его в пробирке. Сопротивление нагревателя составило 80,1 Ом. Для питания нагревателя использовался стабилизированный источник постоянного тока GPR-7550D, напряжение

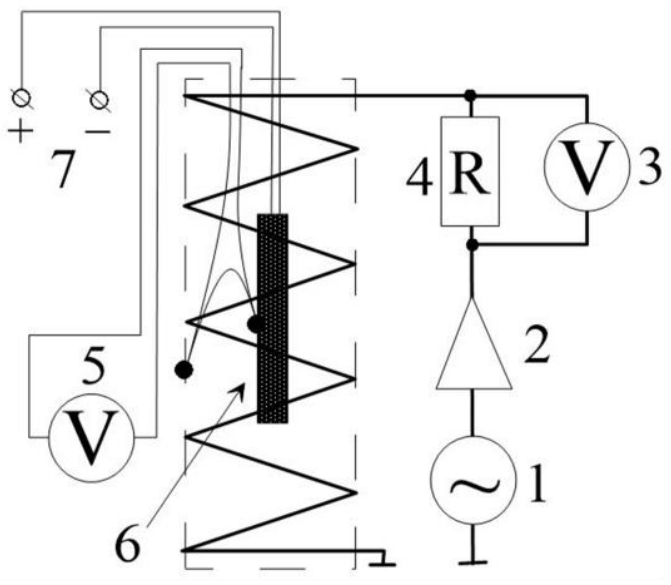

Рис. 2. Схема экспериментальной установки в градуировочном варианте: 1-генератор

ГЗ-112/1, 2-усилитель 100У-101, 3-вольтметр Ф5053, 4-безындуктивный резистор, 5-вольтметр Щ300,

6-пробирка с нагревателем, 7-выходные клеммы источника тока GPR-7550D

на выходных клеммах которого измерялось вольтметром GDM-8246.

Мощность нагревателя изменялась ступенчато, а стационарный температурный режим достигался через 1-1,5 ч после её изменения. Для максимального соответствия условий градуировки условиям опытов с магнитной жидкостью, через обмотку соленоида пропускался переменный ток той же силы, что и в реальных опытах. Примеры градуировочных кривых $\varepsilon(Q)$ для двух значений силы тока в соленоиде представлены на рис. 3 .

Как видно, полученные зависимости $\varepsilon(Q)$ хорошо аппроксимируются линейными функциями.

Основная систематическая ошибка, возникающая при вычислении мнимой восприимчивости $\chi_{2}$ по тепловыделению, 


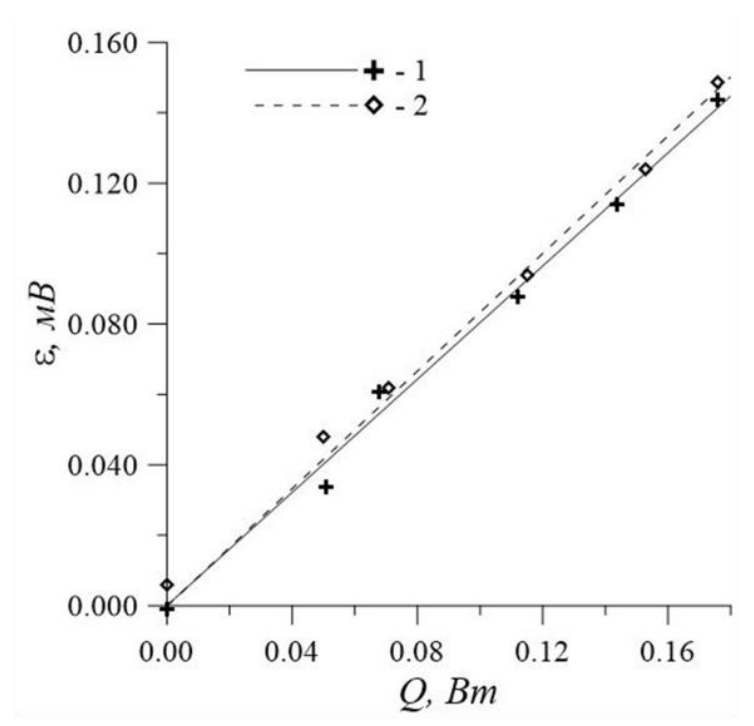

Рис. 3. ЭДС термопары в зависимости от мощчности электронагревателя для двух значений силь тока в соленоиде 1-0,20 A, 2-0,29 А. Точками обозначень экспериментальные значения, прямыми - результат линейной аппроксимации

связана с её температурной зависимостью и изменением температуры магнитной жидкости вслед за изменением частоты и амплитуды поля. Для контроля температуры жидкости использовалась дополнительная медь-константановая термопара, горячий спай которой находился непосредственно в пробирке, а холодный -

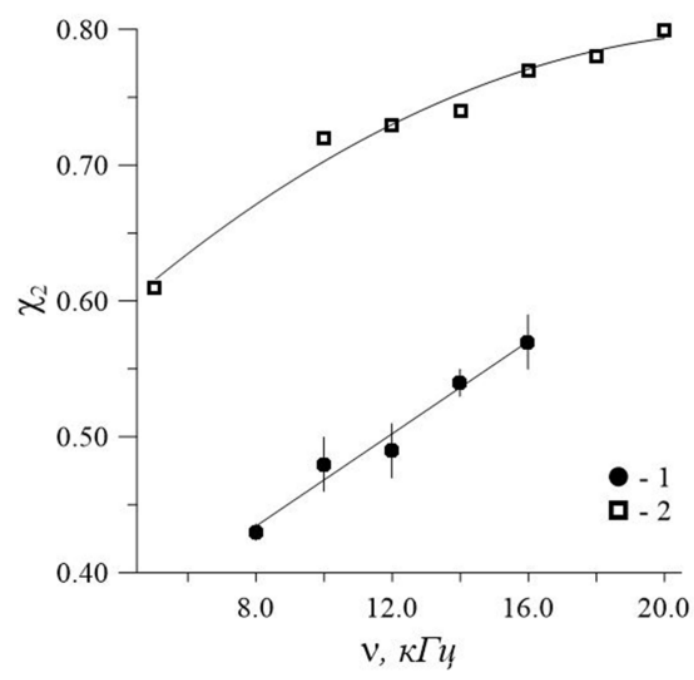

в сосуде с тающим льдом. Контрольные зависимости $\chi_{2}$ от температуры $t$ определялись из результатов измерений мостом взаимной индуктивности в пределе малых амплитуд [7]. Полученные зависимости $\chi_{2}(t)$ аппроксимировались линейными функциями в пределах температур, достигаемых в опытах с тепловыделением.

Результаты измерений мнимой части динамической восприимчивости по тепловыделению магнитной жидкости при повышенных амплитудах магнитного поля (до 1,3 кА/м) сравнивались с результатами измерений мостом взаимной индуктивности при малых амплитудах (до 200 А/м). При сравнении вносились поправки, связанные с объёмным расширением жидкости при увеличении температуры. Результаты сравнения приведены на рис. 4 для частот зондирующего поля 10 и 12 кГц.

Эти результаты выглядят вполне ожидаемыми. Наблюдается незначительное (в пределах 1-5\%) уменьшение мнимой части восприимчивости с ростом амплитуды поля. По нашему мнению, такое поведение динамической восприимчивости связано с предварительным удалением из жидкости крупнодисперсной фракции. В результате

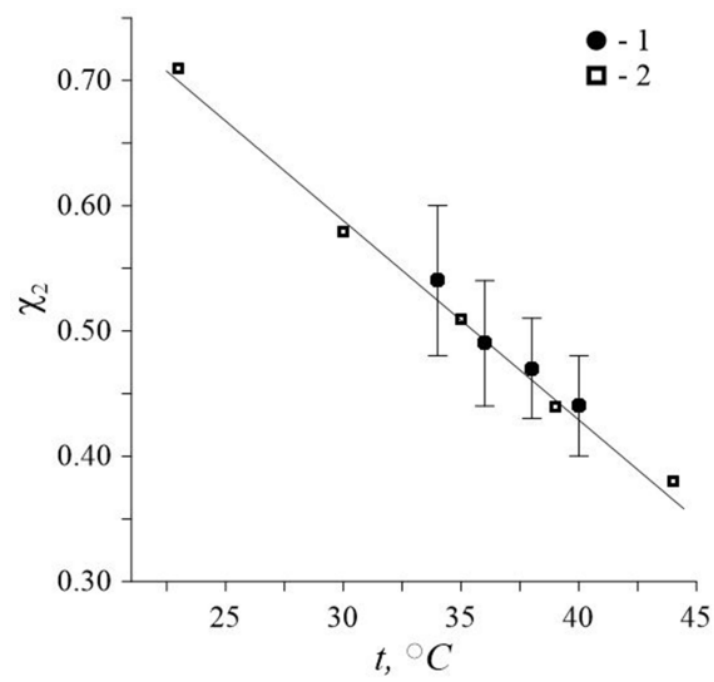

Рис. 4. Мнимая часть восприимчивости в зависимости от температуры для частот зондирующего поля 10 и 12 кГи соответственно: 1 - расчёт по мощности тепловыделения, 2 - измерение мостом взаимной индуктивности.

Прямыми показана линейная аппроксимаџия результатов, полученных с использованием моста взаимной индуктивности 
преобладающими оказываются фракции частиц, для которых параметр Ланжевена (6) остаётся малым и при повышенных амплитудах поля. Как следствие, выполняются и формулы (5) для спектральных амплитуд.

Частотные зависимости мнимой части восприимчивости строились из результатов измерений по тепловыделению и мостом взаимной индуктивности (рис. 5).

Увеличение $\chi_{2}$ с ростом частоты означает, что максимум мнимой восприимчивости соответствует более высоким, чем ожидалось, частотам. Данный результат выглядит вполне естественным, так как присутствие в растворе большого числа мелких частиц, в том числе обладающих неелевским механизмом релаксации, не было учтено при выборе диапазона рабочих частот.

В целом, полученные нами результаты позволяют предположить, что амплитудная зависимость динамической восприимчивости магнитной жидкости с малой концентрацией крупных частиц на звуковых частотах оказывается слабой. Слагаемые, соответствующие наиболее крупным частицам (4), существенно зависят от амплитуды поля (при фиксированной частоте), но их вклад

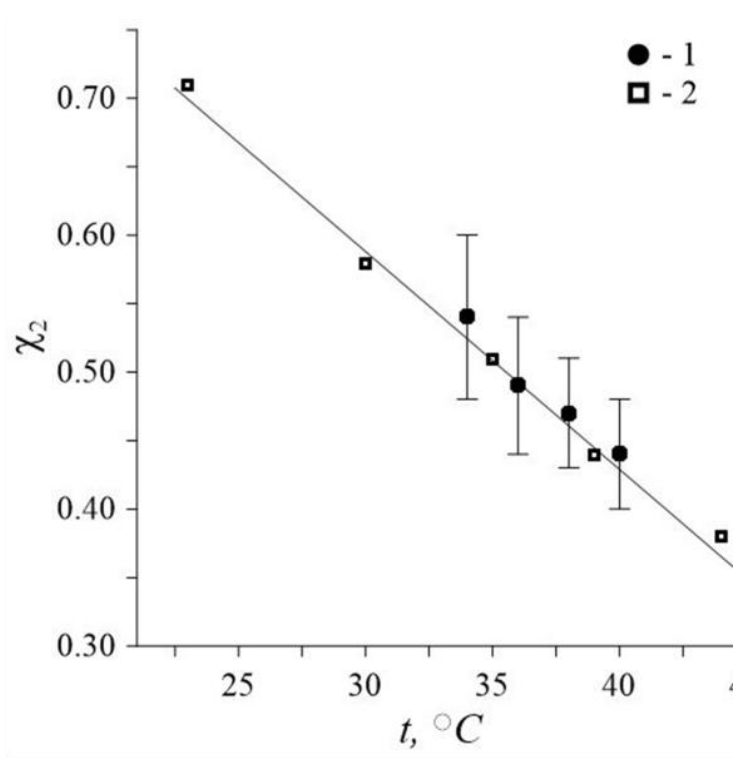

Рис. 5. Мнимая часть динамической восприимчивости в зависимости от частоть зондирующего поля: 1-расчёт по мощности тепловыделения, 2-результат измерений мостом взаимной индуктивности при комнатной температуре. Точки соответствуют экспериментальным данным, кривые - аппроксимации полиномом второго порядка

в восприимчивость остаётся незначительным. Таким образом, область магнитных полей, при которых справедливо уравнение (3) на звуковых частотах, может быть расширена по крайней мере до 1,3 кА/м.

\section{Библиографический список}

1. Блум Э.Я., Майоров М.М., Цеберс А.О. Магнитные жидкости. - Рига: Зинатне, 1989. - 386 с.

2. Бруснецов Н.А., Шевелев А.А., Бруснецова Т.Н. [и др.]. Магнитожидкостная регионарная индукционная гипертермия саркомы // Химико-фармацевтический журнал. - 2002. - Т. 36. - Вып. 3. - С. 8-10.

3. Лахтина Е.В., Пшеничников А.Ф. Дисперсия магнитной восприимчивости и микроструктура магнитной жидкости // Коллоидный журн. - 2006. - Т. 68. - Вып. 3. - С. 1-11.

4. Михайлов Г.А., Васильева О.С. Технология будущего: использование магнитных частиц в онкологии // Бюллетень СО РАМН. - 2008. - Т. 131. - Вып. 3. - С. 8-22.

5. Пиеничников А.Ф., Лебедев А.В. Магнитная восприимчивость концентрированных ферроколоидов // Коллоидный журн. - 2005. - Т. 67. - Вып. 2. - С. 1-13.

6. Пшеничников А.Ф., Лебедев А.В., Радионов А.В., Ефремов Д.В. Магнитная жидкость для работы в сильных градиентных полях // Коллоидный журн. - 2015. - Т. 77. - Вып. 2. - С. 207-213.

7. Пшеничников А.Ф. Мост взаимной индуктивности для анализа магнитных жидкостей // Приборы и техника эксперимента. - 2007. - № 4. - С 88-93.

8. Розенцвейг Р. Феррогидродинамика: пер. с англ. - М.: Мир, 1989. - 356 с.

9. Стародубиев Ю.Н. Магнитомягкие материалы. Энциклопедический словарь-справочник. М.: Техносфера, 2011. - 664 с.

10. Шлиомис М.И. Магнитные жидкости // Успехи физических наук. - 1974. - Т. 112. - Вып. 3. - С. $427-458$.

11. Chow T.S. Viscoelasticity of concentrated dispersions // Physical review E. - 1994. - Vol. 50. - P. 1274. 
12. Ivanov A.O., Kantorovich S.S., Zverev V.S. [et al.]. Temperature-dependent dynamic correlations in suspensions of magnetic nanoparticles in a broad range of concentrations: combined experimental and theoretical study // Physical Chemistry Chemical Physics. - 2016. - Vol. 18. - P. 18342-18352.

13. Ivanov A.O., Kuznetsova O.B. Magnetic properties of dense ferrofluids: an influence of interparticle correlations // Physical Review E. - 2001. - Vol. 64, - N 4. - P. 401-405.

14. Rosensweig R.E. Heating magnetic fluid with alternating magnetic field // Journal of magnetism and magnetic materials. - 2002. - Vol. 252. - P. 370-374.

\title{
ENERGY DISSIPATION IN THE MAGNETIC FLUID UNDER THE ACTION OF THE FIELD OF ACOUSTIC FREQUENCY
}

\author{
M.A. Koskov ${ }^{1,2}$, A.F. Pshenichnikov ${ }^{1,2}$ \\ ${ }^{1,2}$ Institute of Continuous Media Mechanics UB RAS \\ ${ }^{1,2}$ Perm State National Research University
}

The article presents the results of experimental studies of energy dissipation in a magnetic fluid in an alternating magnetic field of the acoustic frequency. The study aims at obtaining the information on the amplitude dependence of the dynamic susceptibility and the limits of applicability of the linear relaxation equation to magnetization. During the experiment a magnetic fluid purified from a coarse fraction by centrifugation was used. The measurements of heat release in a magnetic fluid with increased amplitudes of a magnetic field were carried out. A well-known formula relating the heat release power to an imaginary part of the susceptibility and which directly follows from the linear relaxation equation was used. The control direct measurements of the dynamic susceptibility were carried out with the use of the method of mutual inductance in a weak probing field with different values of the temperature of the magnetic fluid. The comparison of the results obtained by different methods did not reveal a significant discrepancy between the two series of experiments, which is explained by the substantial predominance in the solution of particles for which the Langevin parameter remains small with increased amplitudes of the probe field. As a result, the dynamic susceptibility remains quasi-independent of the field intensity up to at least $1,3 \mathrm{kA} / \mathrm{m}$.

Keywords: alternating magnetic field, energy dissipation, magnetic fluid, dynamic susceptibility.

\section{Сведения об авторах}

Косков Михаил Андреевич, лаборант, Институт механики сплошных сред УрО РАН - филиал Пермского федерального исследовательского центра УрО РАН (ИМСС УрО РАН), 614013, г. Пермь, ул. Академика Королева, 1; студент 4-го курса направления «Прикладные математика и физика», Пермский государственный национальный исследовательский университет (ПГНИУ), 614990, г. Пермь, ул. Букирева, 15; e-mail: koskov.m@icmm.ru

Пиеничников Александр Фёдорович, доктор физико-математических наук, главный научный сотрудник, ИМСС УрО РАН; профессор кафедры фазовых переходов, ПГНИУ; e-mail: pshenichnikov@icmm.ru 